\title{
SPACECRAFT OBSERVATION OF VENUS INFRA-RED LIMB-DARKENING
}

\author{
Par Carl Sagan (1) \\ (Harvard University and Smithsonian Astrophysical Observatory Cambridge, Massachusetts, U. S. A.)
}

and

James B. Polladr

(Harvard College Observatory, Cambridge, Massachusetts U. S. A.)

RESUME. - - On compare les observations de l'assombrissement centre-bord de Vénus avec ce que l'on peut calculer à partir de différents modèles d'atmosphère et de système nuageux. Les observations ont été faites à bord de Mariner II

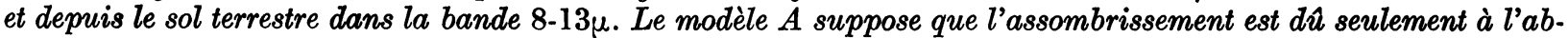
sorption moléculaire dans l'atmosphère de Vénus au-dessus de la couche nuageuse. Le modèle $B$ fait appel à la diffusion multiple par des nuages de particules dont l'albédo et l'altitude sont distribués arbitrairement. Pour chaque modèle on suppose que la distribution des températures est imposée soit par un équilibre convectif, soit par un équilibre radiatif. Dans le modèle $A$, l'assombrissement provient de l'augmentation de la température avec la profondeur. Pour le modèle $B$, la relation $T-\tau$ et la variation de l'émissivité avec l'angle d'émergence contribuent au phénomène. Tous les modèles peuvent être en accord avec les observations moyennant un choix convenable des paramètres et les observations terrestres ne donnent pas d'argument décisif. Mais une sonde spatiale ayant des performances seulement un peu meilleures que Mariner II pourrait déterminer sans ambiguïté la cause de l'assombrissement centre-bord et apporter des informations sur la structure et la composition de l'atmosphère de Vénus.

ABstract. - The limb-darkening laws predicted for several models of the atmosphere and clouds of Venus are compared with limb-darkening observations in the 8.13 $\mu$ interval made from the ground and from Mariner II. Model $A$ attributes the limb-darkening to pure molecular absorption by the atmosphere above the Cytherean cloud layer: Model $B$, to multiple scattering by cloud particles of arbitrary albedo and altitude distribution. In each model, the temperature regime is considered specified, alternatively by convective and by radiative equilibrium. In Model $A$, limb-darkening arises from the increase of temperature with atmospheric depth. In Model $B$, both the $T-\tau$ relation and the dependence of emissivity on the angle of emergence contribute to the limb-darkening. Each model can be made to agree with observation, with an appropriate specification of atmospheric and cloud parameters. With the angular resolution available from the vicinity of the Earth, a discrimination among these models is difficult to achieve. But a Venus flyby or orbiter which performs only somewhat better than Mariner II can potentially determine uniquely the cause of the limb-darkening, and provide significant new information on the structure and composition of the atmosphere and clouds of Venus.

Реяюме. - Сравнены наблюдения потемнения от центра к краю Венеры с тем, что может быть вычислено исходя из различных атмосфиерных моделей и облачной системы. Наблюдения были сделаны на борту Маринер 2 и с земли в полосе 8-13 $\mu$. Модель А предполагает, что потемнение процсходит только от молекулярного поглощения в атмосфере венеры над облачным слоем. Модель в прибегает к многократному рассеянию образами частиц, альбедо и высота которых распределены произвольно. Для каждой модели предположено, что распределение температур налагается или конвективным равновесием, или лучистым равновесием. В модели А потемнеиние

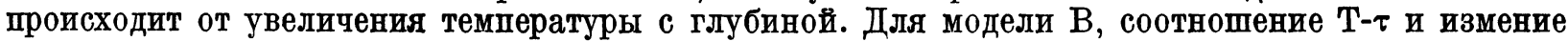
излучательной способности с углом выхождения лучей содействуют явлению. Все модели могут быть в согласии с наблюдениями при надлежащем подборе параметров, а ваземные наблюдения не дают решающего аргумента. Но пространственный зонд с достижениями лишь немного лучпими Маринер 2 смог бы недвусмысленно определить причину потемнения от центра к краю и принести информацию о структуре и составе атмосферы Венеры.

(1) Alfred P. Sloan, Foundation Research Fellow. 
As is true in solar astrophysics, substantial information can be gathered about the outer reaches of a planetary atmosphere by observations of limb-darkening. The problem is complicated by the fact that only close to the limb, where observations are difficult to perform, does the really significant data emerge. Because of diffraction, atmospheric seeing, and detector sensitivity limitations, limb-darkening observations with high topographical resolution can in principle be performed best from a flyby or orbiting spacecraft. In practice, and to date, groundbased observations have retained a slight superiority.

Observations of Venus performed by SINToN and Strong (1960) at the 200-inch Hale reflector through the 8-13 $\mu$ window in the terrestrial atmosphere yield a brightness temperature at the center of the disk of $234 \mathrm{~K}$, and a limb-darkening law which can be expressed as

$$
I_{w}(\mu) \propto \mu^{\alpha},
$$

where $I_{v o}(\mu)$ is the specific intensity, $w$ indicates the 8-13 $\mu$ window, and $\mu$ is the cosine of the angle between the line of sight and the local planetary normal. For $\mu^{-1}$ between 1.0 and 1.7, the data yield $\alpha \simeq 0.50 \pm 0.05$. There is no guarantee that the proportionality can be extrapolated in $\mu^{-1}$.

On 14 December 1962, the United States spacecraft Mariner II observed Venus infrared limbdarkening with a 1.2-inch refractor at a distance of $35000-40000 \mathrm{~km}$ from Venus (Chase, Kaplan, and NeUGEBAdER, 1963). Observations were carried out in two channels, one effectively between 7.8 and $9.0 \mu$; the other effectively between 10.1

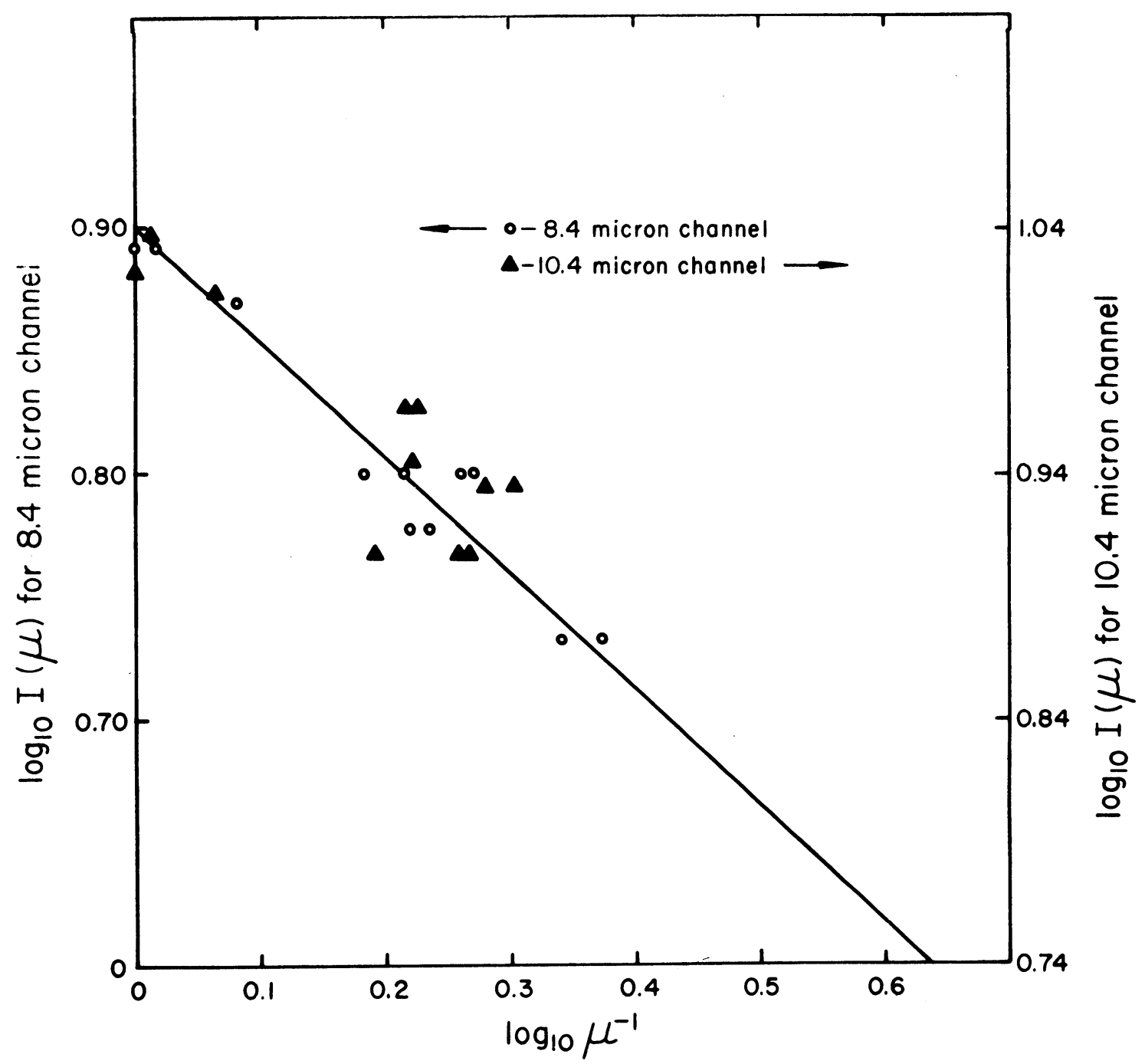

Fia. 1. - Specific intensity observed by Mariner II in the 8.4 and 10.4-micron channels as a function of position over the disk of Venus. High-latitude points have been systematically excluded from the diagram. 
and $10.7 \mu$. The observations for the two channels are plotted separately in Figure 1, from which high-latitude points have been systematically excluded in an effort to remove the effects of a latitudinal temperature gradient at the effective emitting level. For the same reason, all three scans have been plotted together. Over a range in $\mu^{-1}$ between 1.0 and 2.4, the Mariner II data show a limb-darkening law of the form (1), with $\alpha \simeq 0.47 \pm 0.05$. The brightness temperature at the center of the disk was compatible with previous work of SinToN and STRONG, and of others.

At about the same time that Mariner II was passing Venus, observations of Venus limb-darkening were performed from the Earth by MURRAY, WIIDEY, and WeSTPHAL (1963), using, again, the 200-inch Hale telescope, and a mercury-doped germanium photoconductor at liquid helium temperatures. These investigators used a filter transmitting between 8 and $14 \mu$, and although the range in $\mu^{-1}$ observed slightly exceeded that of the previous observations, both the temperatures at the center of the disk $\left(\sim 210^{\circ} \mathrm{K}\right)$ and the exponent of the limb-darkening law

$$
(\alpha \simeq 0.37 \pm 0.05)
$$

were anomalously low. In the following theoretical discussion, we tentatively adopt $\alpha \simeq 0.50$, although extensions to other values of $\alpha$ have been made.

Three general categories of models for the atmosphere and clouds of Venus are here proposed to explain the observed limb-darkening. For each model, (1) convective, and then (2) radiative equilibrium are assumed.

In Models A, limb-darkening is attributed to the temperature gradient in an approximately gray absorber above the visible cloud deck. This unknown absorber causes the atmosphere above the visible clouds to be entirely opaque in the 8-13 $\mu$ interval. If the atmosphere above the clouds is in convective equilibrium.

$$
\frac{\partial \mathrm{T}}{\partial z}=-\frac{g}{\eta c_{p}}
$$

where $g$ is the acceleration due to gravity, $\eta \geqslant 1$ allows for the possibility of release of latent heat by condensation, and $c_{p}$ is the mean specific heat capacity of the atmosphere at constant pressure If we assume that the absorption coefficient in the 8-13 $\mu$ interval, $k_{w} \propto p^{s}$, then for the boundary condition [ $T \rightarrow 0$ as $\tau \rightarrow 0$ ] the equation of radiative transfer may be solved, yielding the observed proportionality (1). The exponent $\alpha=0.5$, provided that $\eta(1+s) \simeq 3.7$, a condition difficult to achieve with familiar materials. Under the same assumptions, and for the boundary condition $\left[\mathrm{T} \rightarrow \mathrm{T}_{0}\right.$ as $\left.\tau \rightarrow 0\right]$, Model Al agrees with observation, provided that $z_{\tau=1} \simeq 3.3 \mathrm{~km} . \quad z_{\tau-1}$ is defined by the equation

$$
\tau=k_{w} \rho_{0} z_{\tau=1}=1,
$$

where $k_{w} \neq k_{w}(z)$, and $\rho_{0}$ is the density at $\tau=0$ Thtus $z_{\tau=1}$ is the distance in which the optical depth changes significantly.

In Model A2, the unknown absorber is uni- formly mixed in an atmosphere in radiative equilibrium, and the Eddington approximation, the condition of constant net flux, and the boundary condition $\left[\mathrm{T} \rightarrow \mathrm{T}_{0}\right.$ as $\left.\tau \rightarrow 0\right]$ are used to solve the equation of transfer. Agreement with observation is secured, provided the opacity in the 8-13 $\mu$ region is about 2.5 times the Rosseland mean opacity, a departure still consistent with the spirit of the graybody approximation. The model predicts, therefore, that the brightness temperature of Venus should be larger than $234{ }^{\circ} \mathrm{K}$ at wavelengths longer than $14 \mu$; it also predicts a planetary bolometric albedo of about 0.67 , in approximate agreement with observation.

In Models B, limb-darkening is attributed to a combination of the temperature gradient and the angular dependence of the emissivity in approximately gray, multiply-scattering clouds initially with isotropic phase functions. Solutions of the equations of radiative transfer are carried out for a wide range of values of $\pi_{0}$, the albedo for single scattering. In Model B1, convective equilibrium is assumed; the extinction coefficient per unit mass is independent of altitude ; and the equations solved, subject to the boundary condition that $\left[p \rightarrow p_{0}\right.$ as $\left.\tau \rightarrow 0\right]$. The predicted limbdarkening laws agree with observation for any given $\pi_{0}$, for certain values of $z_{\tau-1}$, and of $\mathrm{T}_{t}$ and $\mathbf{T}_{l} . \quad \mathrm{T}_{t}$ is the thermometric temperature at the top of the clouds; $\mathrm{T}_{l}$ is the thermometric temperature at the deepest level in the clouds making an effective contribution to the observed emission :

$$
\tau_{l} \simeq \frac{4}{3}\left(1-\pi_{0}\right)^{-\frac{1}{2}}
$$

Now $z_{\tau=1}$ is a measure of the diffuseness of the clouds.

Fro $\eta=1$, appropriate solutions are obtained for $0 \leqslant \pi_{0} \leqslant 0.95$, provided $z_{\tau=1} \simeq 3.3 \mathrm{kms}$. For this range of $\pi_{0}$, the values of $T_{t}$ are all less 
than $220 \mathrm{oK}$, but the values of $T_{l}$ range up to 320 oK. For $\pi_{0} \geqslant 0.99$, the clouds become much more compact $\left(z_{\tau-1} \leqslant 1 \mathrm{~km}\right)$, approaching terrestrial clouds, for which $z_{\tau-1} \sim 50$ meters. For such high single-scattering albedos, the temperatures at the tops of the clouds become $>260{ }^{\circ} \mathrm{K}$; and at the bottoms, $>345 \circ \mathrm{K}$. These combinations of the single-scattering albedo and the temperature range over which the cloud must be stable restrict the variety of conceivable cloud compositions.

In Model B2, the temperature gradient within the clouds is determined by radiative equilibrium and the equations of radiative transfer solved, again, in the Eddington approximation, assuming constant net flux, and subject to the boundary condition that $\left[T \rightarrow T_{0}\right.$ as $\left.\tau \rightarrow 0\right]$. In the case that $0 \leqslant \pi_{0} \leqslant 0.95$, agreement with observation is secured if the 8-13 $\mu$ opacity is about 2.5 times the Rosseland mean opacity. The temperatures within the clouds are comparable to those of Model Bl. The predicted bolometric albedo lies between 0.67 and 0.61 , which is within the range of observational bolometric albedos quoted in the literature. For $\pi_{0} \geqslant 0.99$, the predicted bolometric albedo is $\leqslant 0.13$, inconsistent with observations.

Extensions of the preceding models are made to other values of $\alpha$, and to arbitrary phase functions. It is found that for sufficiently high values of $\pi_{0}$, any given anisotropic phase function reduces for this problem, to the solution for the isotropic phase function.

In Models C, the observed emission is attributed to multiple coherent scattering in the clouds and consequent angular redistribution of radiation incident from the atmosphere below. The model requires high values of the single-scattering albedo within the clouds; but in addition, since the observed radiation must come from a considerable depth below the clouds, the 9.4 and $10.4 \mu$ $\mathrm{CO}_{2}$ absorption bands should be present in the spectrum of Venus, contrary to observation. Thus, Model $\mathrm{C}$ is rejected.

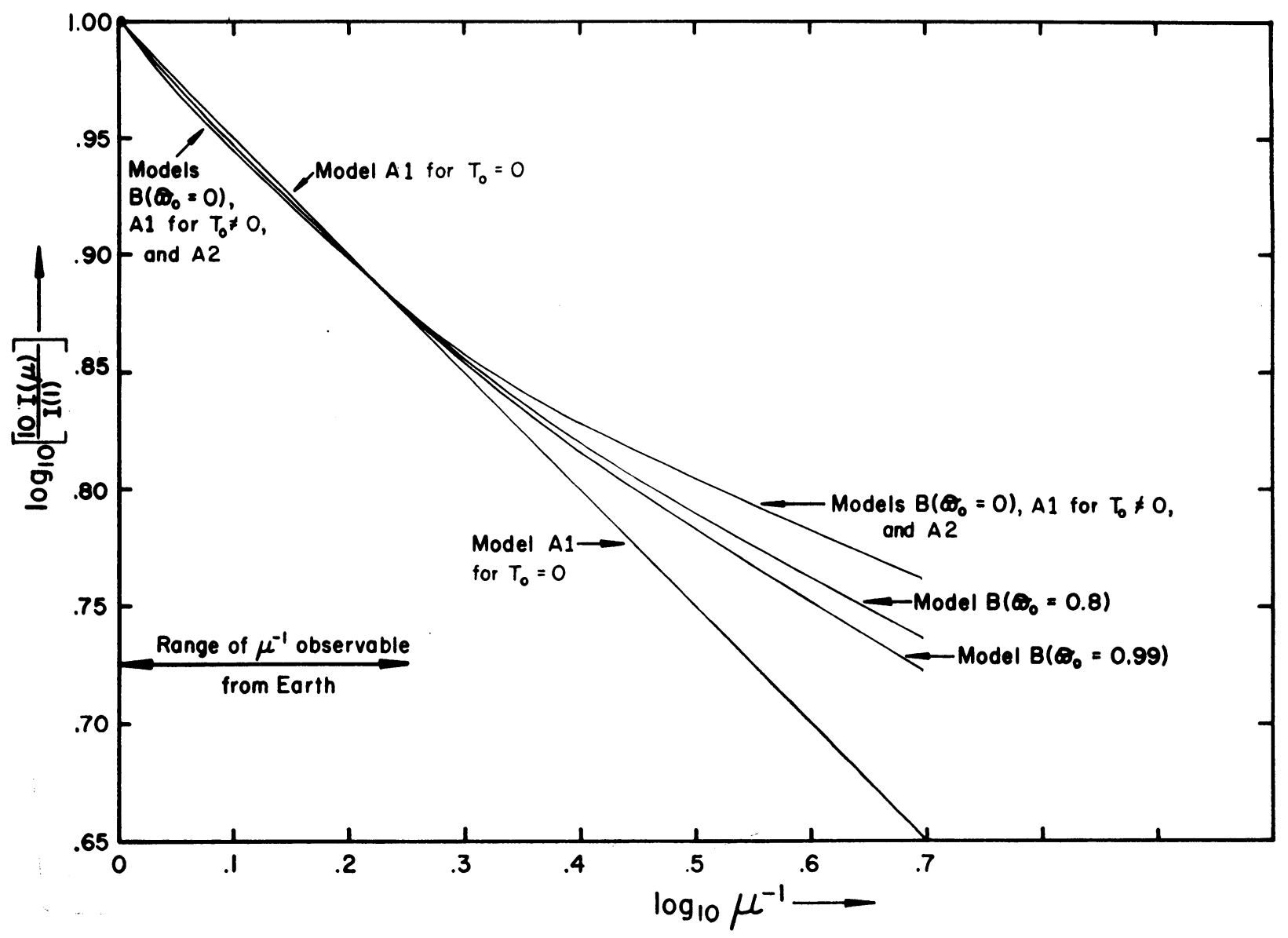

Fra. 2. - Predioted limb-darkening laws for a variety of possible models of the atmosphere and clouds of Venus. All the models match the observations over the range in $\mu$ observed from Earth (see text for details). 
The limb-darkening predicted by the surviving models are displayed in Figure 2. We note that over the range of $\mu^{-1}$ observable from the Earth, all models give the observed limb-darkening law ; but just slightly closer to the limb of Venus, the models diverge markedly, and distinction among them can be made by high topographical resolution limb-darkening observations. In addition, the models predict different limb-darkening laws at other frequencies, permitting them to be distinguished from each other. Limb-darkening observations with high topographical resolution and a wide frequency range are natural experiments for a flyby or orbiter spacecraft in the vicinity of Venus, and provide some justification for further scientific exploration of Venus in the tradition of Mariner 2.

A more detailed account of the discussion of the present paper can be found elsewhere (Pollack and SAGAN, 1965).

Manuscrit reçu le 3 octobre 1964.

\section{REFERENCES}

Chase S. C., Kaplan L. D. and Neugebauer G., 1963, J. Geophys. Res., 68, 6157.

MURray B. C., Wildey R. L. and Westphal J. A., 1963, J. Geophys. Res., 68, 4813.
Pollack J. B. and SagaN C., 1965, to be published. Sinton W. M. and Strona J., 1960, Ap. J., 131, 470.

\section{Discussion}

L. H. Allur. - Did not SpINRAD's observations of infrared spectrum of Venus suggest that transparency of atmosphere changes with time ? Different rotational temperatures were observed at different times.

C. Sagan. - Yes. These observations (H. SpInRAD, Publications of the Astronomical Soc. of the Pacific, 74, 187, 1962) indicated a correlation of the rotational temperature with line contour pressure in the $7820 \AA$ Venus $\mathrm{CO}_{2}$ band. High temperatures accompanied high pressures ; the most likely explanation is that the cloud layer has a variable transparency at this wavelength, and that some days we see to quite considerable depths in the Cytherean atmosphere.

In a paper in press (C. SagaN and J. B. PollaCk, "On the nature of the clouds and the origin of the surface temperature of Venus n), PoLLAOK and I have interpreted the near infrared transparency of the Venus clouds by preferential forward scattering in cloud particles with low indices of refraction, high single scattering albedos, and sizes suggested by the existing polarimetric observations. The asymetric scattering functions required are consistent with those deduced from the brightness phase function of Venus at optical frequencies. 